\title{
Exploring the Inner Narrow-Line Regions of Seyfert Galaxies
}

\author{
S. B. Kraemer ${ }^{1}$, D. M. Crenshaw ${ }^{2}$, M. L. Trippe ${ }^{2}$, \\ H. R. Schmitt ${ }^{3}$, and M. Meléndez ${ }^{4}$ \\ ${ }^{1}$ The Catholic University of America, Washington, DC 20064, USA \\ ${ }^{2}$ Georgia State University, Atlanta, GA 30303, USA \\ ${ }^{3}$ Naval Research Laboratory, Washington, DC 20375, USA \\ ${ }^{4}$ NASA Goddard Space Flight Center, Greenbelt, MD 20771, USA
}

Keywords. galaxies: Seyfert, galaxies: individual (Mrk 573), line: formation

In HST/STIS optical spectra of Seyfert galaxies, there is often a bright knot of [O III] emission in the inner NLR. In the case of the Seyfert 1 galaxy NGC 4151, we have shown that the emissionline gas may be associated with the mass outflow detected in absorption in UV spectra, which suggests that we are probing regions close to the AGN. Here we present results for the luminous Seyfert 2 galaxy Mrk 573. The spectra reveal the presence of lines from a wide range of ionization states (see Figure 1), which is indicative of the heterogeneous nature of the emission-line gas. As in our studies of other Seyfert galaxies, including NGC 1068, NGC 4151, and Mrk 3, there is evidence for emission from low-ionization gas outside the nominal emission-line bicone. Finally, based on photoionization modeling, we find that the central emission-line knot in Mrk 573 is some tens of parsecs from the AGN, similar to other Seyfert 2 galaxies, which suggests that the material that is obscuring the central AGN in Seyfert 2s must have a similar radial extent.

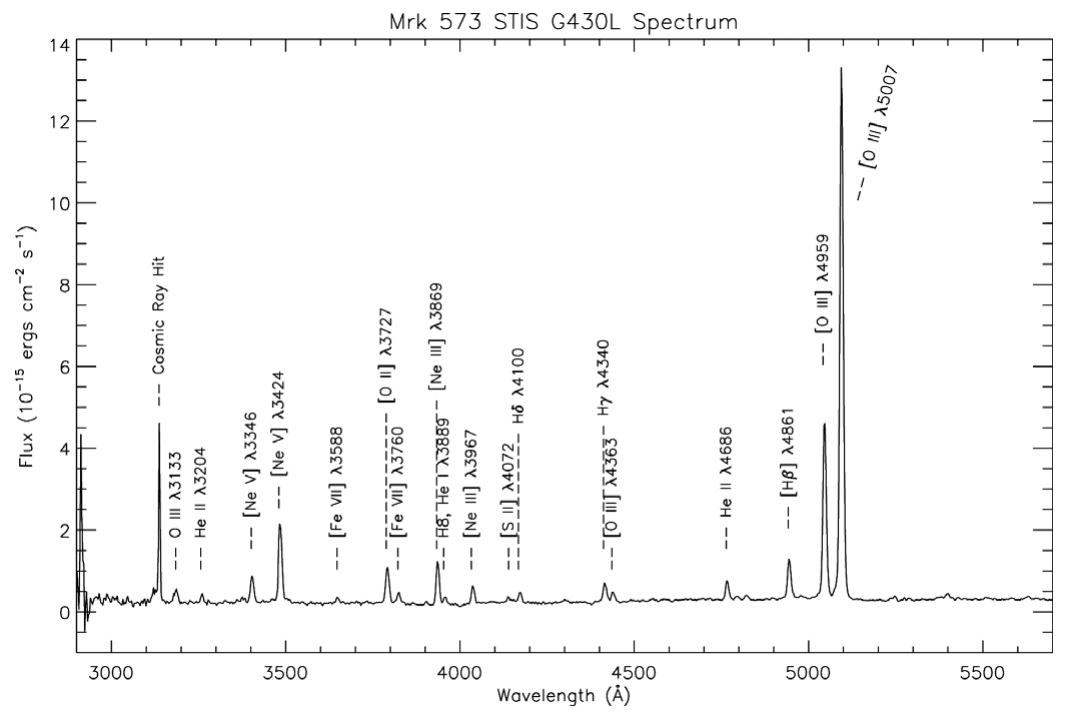

Figure 1. STIS G430L spectrum of the central NLR knot in Mrk 573. 